11. Shlush LI, Zandi S, Mitchell A, Chen WC, Brandwein JM, Gupta $\mathrm{V}$, et al. Identification of pre-leukaemic haematopoietic stem cells in acute leukaemia. Nature. 2014;506:328-33.

12. Gaidzik VI, Weber D, Paschka P, Kaumanns A, Krieger S, Corbacioglu A, et al. DNMT3A mutant transcript levels persist in remission and do not predict outcome in patients with acute myeloid leukemia. Leukemia. 2017;32:30-7.
13. Hollein A, Meggendorfer M, Dicker F, Jeromin S, Nadarajah N, Kern W, et al. NPM1 mutated AML can relapse with wild-type NPM1: persistent clonal hematopoiesis can drive relapse. Blood Adv. 2018;2:3118-25.

14. Schnittger S, Schoch C, Kern W, Mecucci C, Tschulik C, Martelli $\mathrm{MF}$, et al. Nucleophosmin gene mutations are predictors of favorable prognosis in acute myelogenous leukemia with a normal karyotype. Blood. 2005;106:3733-9.

Leukemia (2019) 33:2746-2751

https://doi.org/10.1038/s41375-019-0514-9

Acute lymphoblastic leukemia

\title{
Heritable variation at the chromosome 21 gene ERG is associated with acute lymphoblastic leukemia risk in children with and without Down syndrome
}

Adam J. de Smith $\mathbb{D}^{1} \cdot$ Kyle M. Walsh ${ }^{2,3,4}$ • Libby M. Morimoto ${ }^{5}$ - Stephen S. Francis ${ }^{4,6} \cdot$ Helen M. Hansen ${ }^{7}$. Soyoung Jeon ${ }^{1} \cdot$ Semira Gonseth $^{8}$ - Minhui Chen ${ }^{1} \cdot$ Hanxiao Sun ${ }^{1}$ - Sandra Luna-Fineman ${ }^{9}$. Federico Antillón ${ }^{10}$. Verónica Girón $^{10}$ - Alice Y. Kang ${ }^{5} \cdot$ Ivan Smirnov $^{7} \cdot$ Xiaorong Shao $^{5}$ - Todd P. Whitehead - Lisa F. Barcellos $^{5}$. Kent W. Jolly ${ }^{11}$ - Jasmine Healy ${ }^{12} \cdot$ Caroline Laverdière $^{12} \cdot$ Daniel Sinnett $^{12}{ }^{12} \cdot$ Jeffrey W. Taub $^{13} \cdot$ Jillian M. Birch $^{14}$. Pamela D. Thompson ${ }^{14}$. Maria S. Pombo-de-Oliveira ${ }^{15}{ }^{15} \cdot$ Logan G. Spector $^{16} \cdot$ Andrew T. DeWan $^{17}$. Beth A. Mueller ${ }^{18} \cdot$ Charleston Chiang $^{1} \cdot$ Catherine Metayer $^{5} \cdot$ Xiaomei Ma $^{17} \cdot$ Joseph L. Wiemels $^{1,4}$

Received: 21 February 2019 / Revised: 3 April 2019 / Accepted: 1 May 2019 / Published online: 11 July 2019

(c) The Author(s) 2019. This article is published with open access

These authors contributed equally: Adam J. de Smith, Kyle M. Walsh

These authors jointly supervised this work: Xiaomei Ma, Joseph L. Wiemels

Supplementary information The online version of this article (https:// doi.org/10.1038/s41375-019-0514-9) contains supplementary material, which is available to authorized users.

Adam J. de Smith

desmith@usc.edu

1 Center for Genetic Epidemiology, Department of Preventive Medicine, University of Southern California, Los Angeles, CA, USA

2 Department of Neurosurgery, Duke University, Durham, NC, USA

3 Duke Cancer Institute, Duke University, Durham, NC, USA

4 Department of Epidemiology and Biostatistics, University of California San Francisco, San Francisco, CA, USA

5 School of Public Health, University of California Berkeley, Berkeley, CA, USA

6 Division of Epidemiology, School of Community Health Sciences, University of Nevada, Reno, NV, USA

7 Department of Neurological Surgery, University of California San Francisco, San Francisco, CA, USA

8 Division of Chronic Disease, Institute of Social and Preventive

\section{To the Editor:}

Children of Latino ancestry have $~ 1.6$-fold increased risk of acute lymphoblastic leukemia (ALL) relative to non-Latino white children [1], partly explained by the higher frequency

Medicine, Lausanne, Switzerland

9 Children's Hospital Colorado and University of Colorado, Denver, CO, USA

10 Unidad Nacional de Oncología Pediatrica; and Universidad Francisco Marroquín, Guatemala City, Guatemala

11 Department of Pediatrics, Kaiser Permanente, Roseville, CA, USA

12 Sainte-Justine University Health Center, Montreal, QC, Canada

13 Division of Hematology/Oncology, Children's Hospital of Michigan, Wayne State University School of Medicine, Detroit, MI, USA

14 University of Manchester, Manchester, UK

15 Instituto Nacional de Cancer, Rio de Janeiro, Brazil

16 Department of Pediatrics, University of Minnesota, Minneapolis, MN, USA

17 Department of Chronic Disease Epidemiology, Yale School of Public Health, New Haven, CT, USA

18 Fred Hutchinson Cancer Research Center, Seattle, WA, USA 
of common heritable ALL risk alleles at ARID5B, GATA3, and PIP4K2A in Latinos [2-4]. However, the etiologies of the increased ALL risk in Latinos have not been fully elucidated. We previously performed a large, multi-ethnic genome-wide association study (GWAS) of childhood ALL, including 3,263 cases of which $~ 60 \%$ were of Latino ethnicity [5]. While we identified two novel risk loci, we did not identify Latino-specific risk loci, unlike a recent report from Qian et al. [6]. We have performed whole-genome imputation of our Latino dataset and combined it with GWAS data from two additional, non-overlapping Latino childhood ALL case-control datasets to identify novel and/ or Latino-specific risk loci.

The GWAS meta-analysis included the following: (i) 1,949 ALL cases and 2,120 controls from the California Cancer Records Linkage Project (CCRLP-LAT) study, supplemented with 6464 Kaiser GERA study controls [5]; (ii) 38 cases and 49 controls from a Guatemalan ALL case-control study (GTM); and (iii) 312 cases and 454 controls from the California Childhood Leukemia Study (CCLS) [7] (Supplementary Material). Methods for haplotype phasing, whole-genome imputation, and quality-control of imputed genotypes are described in Supplementary Material. Case-control association analyses were performed separately in each study using logistic regression in SNPTEST V2, adjusting for ten ancestry-informative principal components, calculated separately within each dataset. Within-study genomic inflation factors were low $\left(\lambda_{\mathrm{CCRLP}}=1.034, \lambda_{\mathrm{GTM}}=1.01\right.$, $\lambda_{\text {CCLS }}=1.025$ ). A fixed-effects meta-analysis was performed, and QQ plots indicated adequate control of type I error and minimal population stratification $\left(\lambda_{\text {Meta }}=1.029\right)$ (Supplementary Fig. S1).

Our GWAS meta-analysis of 2,299 cases and 9,087 controls (Latino only) identified genome-wide significant associations $\left(P<5.0 \times 10^{-8}\right)$ at seven well-established risk
Fig. 1 Novel ALL GWAS association locus at $E R G$ on chromosome 21. a Manhattan plot displaying genome-wide $-\log _{10}(P)$ values from a metaanalysis of three Latino ALL GWAS ( $n=2,299$ cases and 9,087 controls), in the California Cancer Records Linkage Project (CCRLP), the California Childhood Leukemia Study (CCLS), and a Guatemalan ALL study. Grey horizontal line represents the genome-wide significance threshold of $P=$ $5.0 \times 10^{-8}$. Genome-wide significant signals were observed at 7 known loci at, in chromosomal order, IKZF1, CDKN2A, GATA3, BMII, $P I P 4 K 2 A, A R I D 5 B$, and $C E B P E$, as well as a novel locus at $E R G$ on chromosome 21. b Locus Zoom plot of a $\sim 500 \mathrm{~Kb}$ region at chromosome $21 \mathrm{q} 22.2$ encompassing $E R G$. The $-\log 10(P)$ values were calculated from meta-analysis of the three Latino case-control studies. The ALL association peak is flanked by two recombination hotspot peaks, represented by vertical blue lines
A

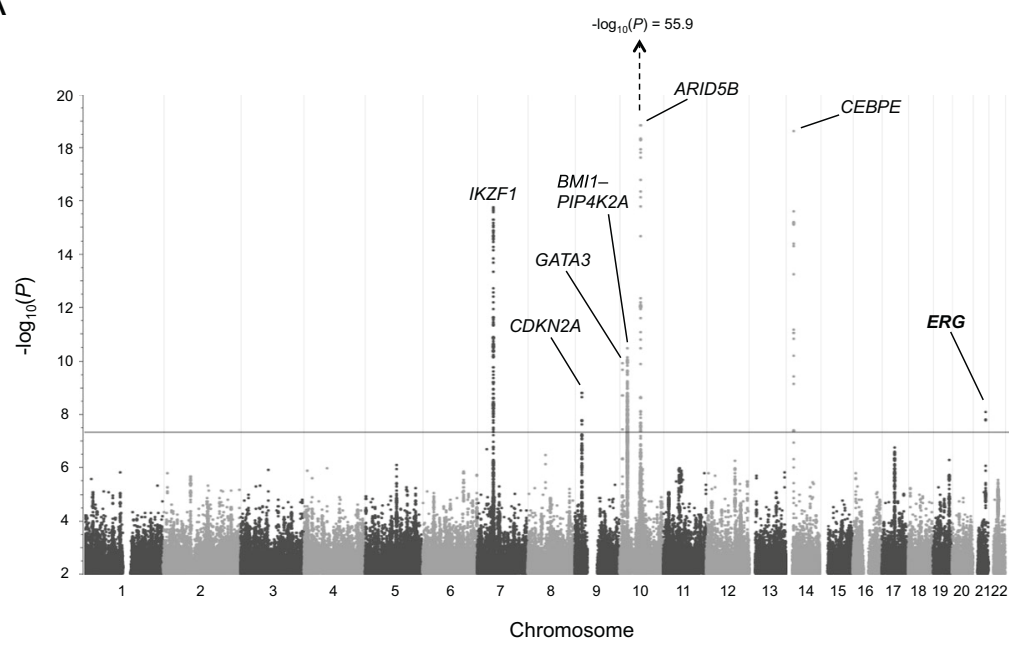

B

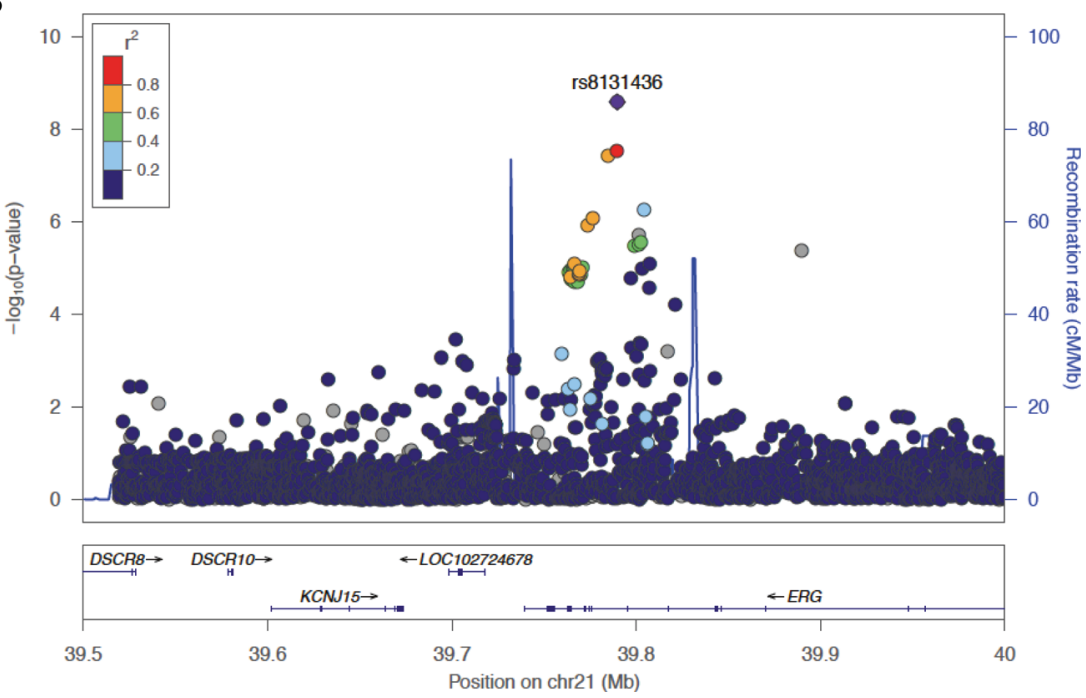


loci at ARID5B, CEBPE, IKZF1, PIP4K2A, GATA3, $C D K N 2 A$, and $B M I 1$ [4, 7-10], plus associations $(P<5.0 \times$ $\left.10^{-4}\right)$ at recently identified loci at $17 \mathrm{q} 12 / I K Z F 3,8 \mathrm{q} 24$, $L H P P$, and ELK3 [5, 11] (Supplementary Table S1). We also identified genome-wide significant association at rs8131436 on chromosome 21q22.2, in an intron of the erythroblast transformation-specific (ETS)-related gene $(E R G)\left(P=8.76 \times 10^{-9}\right.$; odds ratio $[\mathrm{OR}]=1.23 ; 95 \% \mathrm{CI}$ : 1.16-1.31) (Fig. 1a). Targeted re-imputation localized the association to an $\sim 100 \mathrm{~Kb}$ locus between two recombination peaks (Fig. 1b, Supplementary Table S2).

The effect of this locus on ALL risk was recently reported to increase with increasing global Native American (NA) ancestry [6]. Here we examined local ancestry at the ERG locus (Supplementary Material, Supplementary Fig. S2), and found a larger effect size for rs8131436 in Latinos with $\geq 1$ copy of the NA haplotype $(\mathrm{OR}=1.30$; 95\% CI $\left.=1.15-1.47 ; P=2.4 \times 10^{-5}\right)$ than in Latinos with zero NA haplotypes $(\mathrm{OR}=1.15 ; 95 \% \mathrm{CI}=0.98-1.34 ; P=$ 0.09), further supporting a positive association between NA ancestry and the effect of $E R G$ heritable variation on ALL risk. The frequency of NA haplotypes at rs 8131436 was slightly higher in cases (42.7\%) than controls (40.9\%) (Supplementary Fig. S3); however, taking into account the proportion of global NA ancestry, the case-control difference in local NA ancestry at $E R G$ was not significant $(P=0.44)$ (Supplementary Table S3).

Next, we investigated whether any ERG SNPs were associated with ALL risk in non-Latino whites $(n=1184$ cases, 3551 controls from CCRLP-EUR) [5]. Of the top 10 $E R G$ SNPs in our discovery Latino ALL GWAS metaanalysis, SNP rs2836371 was also associated with ALL in non-Latino whites $\left(P=8.40 \times 10^{-3}\right)$, albeit with a smaller effect size $(\mathrm{OR}=1.15,95 \%$ CI: $1.05-1.25)$ (Supplementary Table S2).

$E R G$ is within the Down syndrome (DS) critical region on chromosome 21, and children with trisomy 21 have an 20-fold increased risk of ALL [12]. Therefore, we explored whether $E R G$ variation may contribute to DS-ALL risk. We genotyped rs2836371 (lead SNP across Latino discovery and non-Latino white replication sets) using a Taqman SNP genotyping assay in a Latino case-control set (DS-ALL cases, $n=103$ and DS non-leukemia controls, $n=96$ ) from the International Study of Down Syndrome Acute Leukemia (IS-DSAL, Supplementary Material). Trisomic genotypes were manually clustered to delineate the two heterozygote genotypes (TTC or TCC) (Supplementary Fig. S4). We found that rs2836371 was significantly associated with risk of DS-ALL $(P=0.016)$ with a per-allele OR of 1.44 (95\% CI: 1.08-1.96), which was noticeably but non-significantly higher than that in non-DS Latinos $(\mathrm{OR}=1.19$, Supplementary Table $\mathrm{S} 2)\left(P_{\text {interaction }}=\right.$ $0.21)$. Furthermore, subjects with three risk alleles at

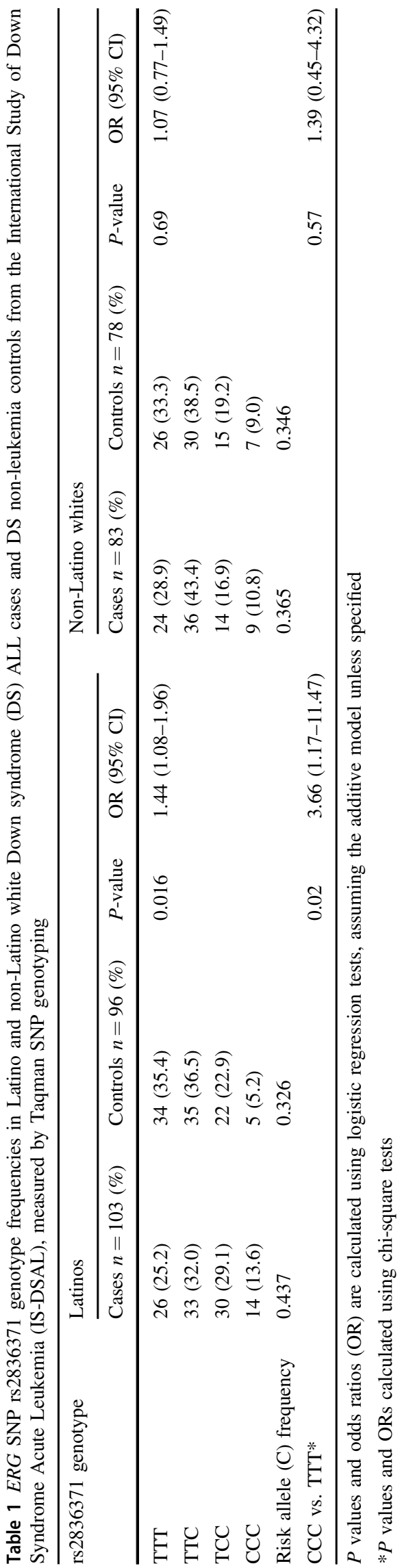


rs2836371 (CCC genotype) had a 3.7-fold increased risk of ALL compared to DS subjects harboring no risk alleles (TTT), rather than the 2.99-fold increased risk predicted under an allelic additive model (Table 1, Supplementary Fig. S5). In a smaller set of non-Latino white DS-ALL cases $(n=83)$ and DS controls $(n=78)$, rs2836371 was not significantly associated with DS-ALL risk $(\mathrm{OR}=1.07,95 \%$ CI: 0.77-1.49), reflecting similar inter-ethnic differences in effect size observed in non-DS participants.

Observed inter-ethnic differences in SNP effect size suggest potential interactions with environmental factors, or with additional germline or somatic genetic alterations. Intriguingly, several published GWAS loci for white blood cell (WBC) traits in adults lie $\sim 50 \mathrm{~Kb}$ downstream of rs2836371 within ERG [13]. These SNPs are in very low linkage disequilibrium (LD) with our ALL-associated SNPs, and are positioned on the other side of a strong recombination peak (Supplementary Fig. S6). Novel analysis of selection signals across $E R G$ in Latinos revealed no evidence of positive selection for ALL risk SNPs, but identified a strongly significant signal (population branch statistic $>99^{\text {th }}$ percentile genome-wide; haplotype statistic $>97^{\text {th }}$ percentile) at the downstream WBC trait locus (Supplementary Fig. S6). SNP rs2836426 showed the strongest selection signal $\left(P=2.2 \times 10^{-4}\right)$ and, though in low LD with ALL risk SNP rs2836371 $\left(D^{\prime}=0.16\right.$ in AMR, 1000Genomes), it is in high LD with several WBC traitassociated SNPs $\left(D^{\prime}=1\right.$ in AMR). No direct association was detected between the low-frequency WBC traitassociated SNPs and ALL risk; however, we found marginally significant synergistic interaction between ALLassociated SNP rs2836371 and three perfectly linked WBC trait SNPs (rs80109907, rs7275212, and rs58030288) on ALL risk in Latinos $(P=0.079, \mathrm{OR}=2.00)$ but not in non-Latino whites $(P=0.48, \mathrm{OR}=0.78)$ (Supplementary Table S4), suggesting Latino-specific cooperation between these two independent trait-associated loci in ALL predisposition.

To explore potential functional effects of ALL-associated SNPs in $E R G$, we assessed 32 SNPs with $P<5.0 \times 10^{-5}$ in the Latino meta-analysis, of which 19 replicated in the European data $(P<0.05)$. ERG protein is expressed at low levels in lymphoblastoid cell lines, which prevented accurate expression quantitative trait locus (eQTL) analysis within Genotype Tissue Expression (GTEx) or GEUVADIS RNASeq datasets. In silico analyses, using Haploreg, RegulomeDB, UCSC Genome Browser, and Epigenome Browser, revealed no protein-coding variants, nor any obvious functional candidates based on overlap with putative regulatory elements and transcription factor binding sites.

A recently identified ALL tumor subtype, "DUX4rearranged ALL", is characterized by somatic $D U X 4$ rearrangements that result in alternative splicing of $E R G$ using an alternative start site at "exon 6 alt" [14]. ALLassociated SNPs at ERG did not alter known DUX4 binding motifs, and TF-binding motif analysis did not reveal any SNPs creating novel DUX4 binding motifs.

We assessed whether any SNPs overlapped ERG exon 6 alt and found that SNP rs2836361, in tight LD with rs2836371 $\left(R^{2}=0.93\right.$ and $D^{\prime}=0.97$ in 1000 Genomes individuals of Mexican ancestry; $R^{2}=0.99$ and $D^{\prime}=0.99$ in Europeans), was located $3 \mathrm{bp}$ upstream of the first exon 6 alt codon (Supplementary Fig. S7). SNP rs2836361 disrupts a strong exonic splicing silencer (ESS), with the risk allele reducing the score of a silencer motif "TCTCCCAA" [15] from 88.1 (TCTGCCAA containing the rs2836361 protective allele) to 70.9 (TCTGTCAA containing the risk allele). This ESS had the highest predicted score within a region encompassing exon 6 alt $+/-100 \mathrm{bp}$. Moreover, we found that the rs2836361 risk allele may increase exonic splicing enhancer activity by elevating the RNA recognition motif score for serine/arginine-rich pre-mRNA splicing factor (SRp40). Hence, the rs2836361 risk allele may increase splicing of the non-canonical $E R G$ exon 6 alt, conferring dominant negative effects on wildtype ERG and increased risk of ALL. Further analysis is needed to confirm the causal variant at this locus and its functional effects.

In sum, we report the largest GWAS of childhood ALL among Latinos to date, identifying a risk locus at chromosome 21q22.2, encompassing the hematopoietic transcription factor $E R G$. This gene is frequently somatically mutated in ALL, adding to a growing list of genes that both predispose to ALL and drive tumorigenesis following somatic mutations. Insufficient patient data were available to investigate the relationship between ERG SNPs and somatic alterations; however, during preparation of this manuscript, Qian et al. reported that the ERG risk genotype was negatively correlated with somatic $E R G$ deletions [6], supporting that the SNP may somewhat mimic effects of somatic loss of ERG.

Novel to our study, we replicated the $E R G$ association in a case-control study of Down syndrome-ALL; this is the first reported heritable risk factor for DS-ALL, and may inform future risk stratification in this vulnerable population. Current methods to accurately assess trisomic genotypes using SNP arrays are sub-optimal; next-generation sequencing strategies are warranted to elucidate the contribution of heritable variation across chromosome 21 to DS-ALL risk.

Our study highlights the importance of Latino subjects in elucidating the germline genetic architecture of childhood ALL, and suggests that larger sample sizes may reveal additional important susceptibility loci that inform the biology of leukemogenesis. 


\section{Disclaimer}

The ideas and opinions expressed herein are those of the author (s) and do not necessarily reflect the opinions of the State of California, Department of Public Health, the National Cancer Institute, and the Centers for Disease Control and Prevention or their Contractors and Subcontractors.

Acknowledgements For recruitment of subjects enrolled in the California Childhood Leukemia Study (CCLS), the authors gratefully acknowledge the clinical investigators at the following collaborating hospitals: University of California Davis Medical Center (Dr. Jonathan Ducore), University of California San Francisco (Drs. Mignon Loh and Katherine Matthay), Children's Hospital of Central California (Dr. Vonda Crouse), Lucile Packard Children's Hospital (Dr. Gary Dahl), Children's Hospital Oakland (Drs. James Feusner and Carla Golden), Kaiser Permanente Roseville (formerly Sacramento) (Drs. Kent Jolly and Vincent Kiley), Kaiser Permanente Santa Clara (Drs. Carolyn Russo, Alan Wong, and Denah Taggart), Kaiser Permanente San Francisco (Dr. Kenneth Leung), Kaiser Permanente Oakland (Drs. Daniel Kronish and Stacy Month), California Pacific Medical Center (Dr. Louise Lo), Cedars-Sinai Medical Center (Dr. Fataneh Majlessipour), Children's Hospital Los Angeles (Dr. Cecilia Fu), Children's Hospital Orange County (Dr. Leonard Sender), Kaiser Permanente Los Angeles (Dr. Robert Cooper), Miller Children's Hospital Long Beach (Dr. Amanda Termuhlen), University of California, San Diego Rady Children's Hospital (Dr. William Roberts), and University of California, Los Angeles Mattel Children's Hospital (Dr. Theodore Moore). The authors additionally thank the families for their participation in the CCLS (formerly known as the Northern California Childhood Leukemia Study). The IS-DSAL study included biospecimens and/or data obtained from the California Biobank Program, (SIS requests \# 26 and 572), Section 6555(b), 17 CCR. The California Department of Public Health is not responsible for the results or conclusions drawn by the authors of this publication. The authors would like to thank Robin Cooley and Steve Graham (Genetic Disease Screening Program, California Department of Public Health) for their assistance and expertise in the procurement and management of DBS specimens. We are grateful to the Washington State Department of Health for additional specimen/data access and to William O'Brien of the University of Washington for programming/data management. We are also grateful to the New York State Department of Health Newborn Screening Program, the New York State Cancer Registry, and the New York State Congenital Malformations Registry for additional specimen/data access, and to Drs. Maria Schymura of the NYS Cancer Registry, Marilyn Browne of the NYS Congenital Malformations Registry and Denise Kay of the NYS Newborn Screening Program for case identification, linkage and assistance in the procurement of de-identified DBS specimens and data. This study used biospecimens from the California Biobank Program. Any uploading of genomic data and/or sharing of these biospecimens or individual data derived from these biospecimens has been determined to violate the statutory scheme of the California Health and Safety Code Sections 124980(j), 124991(b), (g), (h), and 103850 (a) and (d), which protect the confidential nature of biospecimens and individual data derived from biospecimens. Certain aggregate results may be available from the authors by request.

Funding This study was supported by Alex's Lemonade Stand Foundation "A" Awards (A.J.D., K.M.W.), the Emerging Investigator Fellowship Grant from the Pediatric Cancer Research Foundation (A.J.D.), The Children's Health and Discovery Initiative of Translating Duke Health (K.M.W.), and research grants from the National Institutes of Health (R01 CA155461 to J.L.W. and X.M., R01 ES009137 to
C.M., P24ES004705 to C.M., R24ES028524 to C.M. and L.M., and R21 ES021819 to L.M.). The 2016-2019 CLIC Scientific Annual Meetings were supported by the National Institute of Environmental Health Sciences of the National Institutes of Health under Award Number U13ES026496. The content is solely the responsibility of the authors and does not necessarily represent the official views of the National Institutes of Health. The collection of cancer incidence data used in the CCRLP study was supported by the California Department of Public Health pursuant to California Health and Safety Code Section 103885; Centers for Disease Control and Prevention's (CDC) National Program of Cancer Registries, under cooperative agreement 5NU58DP003862-04/DP003862; the National Cancer Institute's Surveillance, Epidemiology and End Results Program under contract HHSN261201000140C awarded to the Cancer Prevention Institute of California, contract HHSN261201000035C awarded to the University of Southern California, and contract HHSN261201000034C awarded to the Public Health Institute.

\section{Compliance with ethical standards}

Conflict of interest The authors declare that they have no conflict of interest.

Publisher's note: Springer Nature remains neutral with regard to jurisdictional claims in published maps and institutional affiliations.

Open Access This article is licensed under a Creative Commons Attribution 4.0 International License, which permits use, sharing, adaptation, distribution and reproduction in any medium or format, as long as you give appropriate credit to the original author(s) and the source, provide a link to the Creative Commons license, and indicate if changes were made. The images or other third party material in this article are included in the article's Creative Commons license, unless indicated otherwise in a credit line to the material. If material is not included in the article's Creative Commons license and your intended use is not permitted by statutory regulation or exceeds the permitted use, you will need to obtain permission directly from the copyright holder. To view a copy of this license, visit http://creativecommons. org/licenses/by/4.0/.

\section{References}

1. Dores GM, Devesa SS, Curtis RE, Linet MS, Morton LM. Acute leukemia incidence and patient survival among children and adults in the United States, 2001-2007. Blood. 2012;119:34-43.

2. Xu H, Cheng C, Devidas M, Pei D, Fan Y, Yang W, et al. ARID5B genetic polymorphisms contribute to racial disparities in the incidence and treatment outcome of childhood acute lymphoblastic leukemia. J Clin Oncol. 2012;30:751-7.

3. Walsh KM, Chokkalingam AP, Hsu LI, Metayer C, de Smith AJ, Jacobs DI, et al. Associations between genome-wide Native American ancestry, known risk alleles and B-cell ALL risk in Hispanic children. Leukemia. 2013;27:2416-9.

4. Walsh KM, de Smith AJ, Chokkalingam AP, Metayer C, Roberts $\mathrm{W}$, Barcellos LF, et al. GATA3 risk alleles are associated with ancestral components in Hispanic children with ALL. Blood. 2013;122:3385-7.

5. Wiemels JL, Walsh KM, de Smith AJ, Metayer C, Gonseth S, Hansen HM, et al. GWAS in childhood acute lymphoblastic leukemia reveals novel genetic associations at chromosomes 17q12 and 8q24.21. Nat Commun. 2018;9:286-017-02596-9.

6. Qian M, Xu H, Perez-Andreu V, Roberts KG, Zhang H, Yang W, et al. Novel susceptibility variants at the ERG locus for childhood acute lymphoblastic leukemia in Hispanics. Blood. 2019;133:724-9. 
7. Walsh KM, de Smith AJ, Hansen HM, Smirnov IV, Gonseth S, Endicott AA, et al. A heritable missense polymorphism in CDKN2A confers strong risk of childhood acute lymphoblastic leukemia and is preferentially selected during clonal evolution. Cancer Res. 2015;75:4884-94.

8. Papaemmanuil E, Hosking FJ, Vijayakrishnan J, Price A, Olver B, Sheridan E, et al. Loci on $7 \mathrm{p} 12.2,10 \mathrm{q} 21.2$ and $14 \mathrm{q} 11.2$ are associated with risk of childhood acute lymphoblastic leukemia. Nat Genet. 2009;41:1006-10.

9. Xu H, Yang W, Perez-Andreu V, Devidas M, Fan Y, Cheng C, et al. Novel susceptibility variants at 10p12.31-12.2 for childhood acute lymphoblastic leukemia in ethnically diverse populations. J Natl Cancer Inst. 2013;105:733-42.

10. Trevino LR, Yang W, French D, Hunger SP, Carroll WL, Devidas $\mathrm{M}$, et al. Germline genomic variants associated with childhood acute lymphoblastic leukemia. Nat Genet. 2009;41:1001-5.
11. Vijayakrishnan J, Kumar R, Henrion MY, Moorman AV, Rachakonda PS, Hosen I, et al. A genome-wide association study identifies risk loci for childhood acute lymphoblastic leukemia at 10q26.13 and 12q23.1. Leukemia. 2017;31:573-9.

12. Hasle H, Clemmensen IH, Mikkelsen M. Risks of leukaemia and solid tumours in individuals with Down's syndrome. Lancet. 2000;355:165-9.

13. Astle WJ, Elding $\mathrm{H}$, Jiang $\mathrm{T}$, Allen $\mathrm{D}$, Ruklisa $\mathrm{D}$, Mann AL, et al. The allelic landscape of human blood cell trait variation and links to common complex disease. Cell. 2016; 167:1415-29.

14. Zhang J, McCastlain K, Yoshihara H, Xu B, Chang Y, Churchman ML, et al. Deregulation of DUX4 and ERG in acute lymphoblastic leukemia. Nat Genet. 2016;48:1481-9.

15. Sironi M, Menozzi G, Riva L, Cagliani R, Comi GP, Bresolin N, et al. Silencer elements as possible inhibitors of pseudoexon splicing. Nucleic Acids Res. 2004;32:1783-91.

Leukemia (2019) 33:2751-2753

https://doi.org/10.1038/s41375-019-0516-7

Acute lymphoblastic leukemia

\title{
Humanized CD19-specific chimeric antigen-receptor T-cells in 2 adults with newly diagnosed B-cell acute lymphoblastic leukemia
}

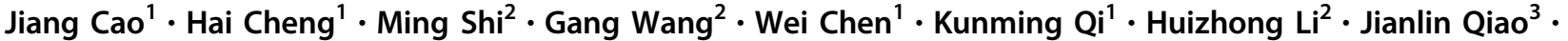 \\ Jing Zhao ${ }^{1} \cdot$ Qingyun $\mathrm{Wu}^{3} \cdot$ Lingyu Zeng $^{3} \cdot$ Guangjun Jing ${ }^{4} \cdot$ Junnian Zheng ${ }^{2,5,6} \cdot$ Kailin Xu $\mathbb{C}^{1,3}$
}

Received: 23 March 2019 / Revised: 5 April 2019 / Accepted: 1 May 2019 / Published online: 15 July 2019

(c) Springer Nature Limited 2019

These authors contributed equally: Jiang Cao, Hai Cheng

Supplementary information The online version of this article (https:// doi.org/10.1038/s41375-019-0516-7) contains supplementary material, which is available to authorized users.

Junnian Zheng

jnzheng@xzmc.edu.cn

Kailin Xu

lihmd@163.com

1 Department of Hematology, The Affiliated Hospital of Xuzhou Medical University, 221002 Xuzhou, China

2 Cancer Institute, Xuzhou Medical University, 221002 Xuzhou, China

3 Jiangsu Bone Marrow Stem Cell Institute, 221002 Xuzhou, China

4 iCARTAB biomedical co. Ltd, 215000 Suzhou, China

5 Jiangsu Center for the Collaboration and Innovation of Cancer Biotherapy, Cancer Institute, Xuzhou Medical University, 221002 Xuzhou, China

6 Center of Clinical Oncology, The Affiliated Hospital of Xuzhou Medical University, 221002 Xuzhou, China

\section{To the Editor:}

Chimeric antigen-receptor T-cell (CAR-T) therapy is safe and effective in advanced B-cell acute lymphoblastic leukemia (BALL) [1-4]. We report using humanized CD19-specific CAR-T (hCART19) to treat two newly diagnosed untreated adults with B-cell ALL. The trial was approved by the Ethics Committee of the Affiliated Hospital of Xuzhou Medical University. Both subjects gave written informed consent.

Subject 1: A 51-year-old female with B-cell ALL was diagnosed in April 2016. The WBC was $2.7 \times 10 \mathrm{E}+9 / \mathrm{L}$ with $1.4 \times 10 \mathrm{E}+9 / \mathrm{L}$ lymphocytes $3 \%$ of which were lymphoblasts. Subset analyses showed $\mathrm{CD}^{+}: 93 \%, \mathrm{CD}^{+}: 54 \%, \mathrm{CD}^{+}$: $24 \%$, and $\mathrm{CD}^{+} 9^{+}: 4 \%$ indicating persisting presumably normal T-cells along with the leukemia B-cells. All lymphoblasts were $\mathrm{CD} 19^{+}$. The bone marrow had $28 \%$ lymphoblasts which were $\mathrm{CD} 19^{+}, \mathrm{CD} 79 \mathrm{a}^{+}, \mathrm{CD} 13^{+}$, and HLA-DR + and weakly positive for $\mathrm{CD} 10, \mathrm{CD} 20$, and $\mathrm{CyCD} 22$. Cytogenetics were normal and BCRABL1 was not detected.

Subject 2: A 69-year-old female diagnosed with B-ALL in February 2017. The WBC was $1.7 \times 10 \mathrm{E}+9 / \mathrm{L}$ with $1 \times 10 \mathrm{E}$ $+9 / \mathrm{L}$ lymphocytes $18 \%$ of which were lymphoblasts. Proportions of $\mathrm{CD}^{+}, \mathrm{CD}^{+}, \mathrm{CD}^{+}$, and $\mathrm{CD} 19^{+}$cells were $68 \%$, 